\title{
Incidence of variations in human cadaveric renal vessels
}

\author{
S.S. Hassan1, 2, E.A. El-Shaarawy², J.C. Johnson³ , M.F. Youakim², R. Ettarh¹ \\ ${ }^{1}$ Department of Medical Education, California University of Science and Medicine, School of Medicine (CalMed-SOM), \\ Colton, California, United States \\ ${ }^{2}$ Anatomy Department, Faculty of Medicine, Cairo University, Cairo, Egypt \\ ${ }^{3}$ A. T. Still Research Institute, A.T. Still University, Kirksville, Missouri, United States
}

[Received: 2 December 2016; Accepted: 6 February 2017]

Background: Awareness of discrepancies of renal vasculature is crucial for some medical procedures. The present study investigated origin and course of aberrant and accessory renal vessels and any associated variations.

Materials and methods: Renal blood vessels of 63 cadavers were examined. Number of renal veins and arteries, arrangement, location where the vasculature attached to the kidneys, and presence of variations were recorded. Incidence of renal vasculature variations was determined, and associations were tested with age at death, sex, and cause of death and whether variations were more common on a specific side.

Results: Variations were found in 7 (11\%; 95\% confidence interval [CI] 5-22\%) cadavers. For renal veins, double, triple, and quadruple veins unilaterally $(5 ; 8 \%)$ and veins that drained the superior pole $(1 ; 2 \%)$ or inferior pole only $(5 ; 8 \%)$ were found. For renal arteries, double and triple arteries unilaterally (3; 5\%) and arteries attached to the superior pole only $(1 ; 2 \%)$ or inferior pole only (2; 3\%) were found. Other variations (polycystic kidney, variations in the common iliac or gonadal veins) were observed. Only renal failure as a cause of death was different between those with or without variations (4/7 [57\%] vs. 1/56 [2\%]; $p<0.001)$. Conclusions: The present study found many variations in renal vasculature. Awareness of such variations may be useful for physicians concerned with this region. (Folia Morphol 2017; 76, 3: 394-407)

Key words: renal vessels, variations, accessory renal artery

\section{INTRODUCTION}

The kidney receives a quarter of the cardiac output that reaches it through the renal artery, which is a branch of the lateral aspect of the abdominal aorta $[18,21]$. The renal artery has many segmental branches supplying different segments of the kidney [21] and an inferior suprarenal branch to the suprarenal gland [18]. This artery is classically considered an end artery that can produce renal ischaemia and infarction in certain renal segments if damaged, obstructed, or ligated by mistake $[18,21]$. The renal veins on both sides of the body connect the hila of both kidneys to the inferior vena cava (IVC). The vein on the right side drains only the right kidney, while the vein on the left side drains the left kidney, left gonads, and left suprarenal gland [21].

Address for correspondence: Dr. E.A. El-Shaarawy, Professor of Anatomy and Embryology, Department of Anatomy and Embryology, Faculty of Medicine, Cairo University, Cairo, Egypt, tel: 011201001582811, e-mail: ehab.elshaarawy@yahoo.com; ehab.elshaarawy@kasralainy.edu.eg 
In a study by Petru et al. [21], a high incidence of variations in renal arteries was found. Other studies have found renal artery variations of number, arrangement, source, and course [10, 29, 33]. In another study, Nayak [18] reported variations in renal vessels associated with variations in the suprarenal and gonadal vessels. Standring [29] suggested that variations in renal arteries are more common than variations in renal veins. A study by Baptista-Silva et al. [2] found variations in the left renal vein (LRV) and double and triple right renal veins (RRVs). Senecail et al. [27] suggested variations of the LRV may be problematic for clinicians interpreting abdominal imaging scans.

With more interventional diagnostic and therapeutic urologic procedures being performed (e.g., renal vessel angiography and angioplasty, renal artery embolisation, or reconstruction of aortic aneurysms), surgeons and radiologists require a deeper appreciation of anatomical variations in the renal vessels $[8,10,16]$. Given the reported variations of the renal vasculature, a better understanding of these variations may minimise accidental bleeding, ischaemia, tubular necrosis, infarcts, rejection, or poor graft function resulting from invasive diagnostic and interventional procedures for the treatment of renal diseases [23]. Therefore, the objective of the present study was to investigate the origin and course of aberrant and accessory renal vessels and any associated variations in the anatomy of this region. By correlating these variations with age at death, sex, and cause of death and with their prevalence on a specific side of the body, we intended to provide more complete information for the surgeons and radiologists treating this important anatomical region.

\section{MATERIALS AND METHODS}

Male and female adult formaldehyde-preserved cadavers were dissected by first-year medical students during the routine dissection of the abdomen, in two consecutive years, following established procedures [30], as part of the Gross Anatomy course at an osteopathic medical school. For the present study, the renal vasculature was specifically investigated. Each year, 5 student volunteers collected and tabulated the data from the dissected cadavers under faculty supervision to ensure the quality of the dissection. The information collected for the present study was neither part of nor did it affect the course grade of the student volunteers. The local institutional review board considered this study as exempt.
The anterior abdominal wall and the abdominal viscera were excised, preserving the retroperitoneal organs, to expose the renal vasculature in the posterior abdominal wall. The number, arrangement, location, and course of the renal vasculature were studied bilaterally, including their connection to the IVC and the abdominal aorta. Other associated variations in the kidneys and surrounding structures were also investigated. The renal vasculature data was recorded on paper, and demographic characteristics of the cadavers were also recorded, including the cadaver number, age at the time of death, sex, and the cause of death. Data about age at death, sex, and cause of death for each cadaver were obtained from the recorded data of the cadaver donation programme. Data about the cause of death was tabulated using the following categories: cardiac (e.g. myocardial infarction, fibrillation, and congestive heart failure), pulmonary (e.g. chronic obstructive pulmonary disease, aspiration pneumonia, and acute respiratory failure), vascular (e.g. stroke, hypertension, embolism, and atherosclerosis), haemorrhagic (e.g. massive bleeding and ruptured aortic aneurism), neurological (e.g. Alzheimer's disease, Amyotrophic Lateral Sclerosis and Parkinson disease), chronic mental illness (e.g. psychosis), primary tumours, metastatic tumours, renal failure, failure to thrive, liver failure, infection, arthritic (e.g. rheumatoid arthritis and hip replacement), and metabolic (e.g. uncontrolled diabetes mellitus). Photographs were taken of the dissected area around the kidneys for only those cadavers with variations in the renal vasculature.

Although various terms are used to describe multiple renal vessels - aberrant, accessory, anomalous, auxiliary, additional, multiple, supernumerary, surplus [13] - we used the term 'accessory' to describe additional renal vessels so we defined "accessory hilar renal artery" as any additional artery separate from the main hilar renal artery and its segmental division, and we defined "accessory polar renal artery" as any artery supplying the kidney at either pole, away from the hilar artery. We also defined an accessory hilar renal vein as any vein that was additional to the usual primary (lobar) tributaries of the hilar renal vein, and we defined an accessory polar renal vein as a vein that was draining separately from either pole of the kidney independently into the IVC, away from the hilar renal vein [24]. Accessory polar vessels can be above or below the usual renal vessels and are connected to the upper or lower poles of the kidneys (superior 
or inferior polar renal vessels, respectively) [33]. We used the term 'aberrant' to describe any vessel in an abnormal anatomical position other than the additional renal vessels such as 'renal artery superficial to renal vein', 'retroaortic renal vein', 'double intercommunicating veins between the two IVC', 'high attachment of gonadal vein to IVC', or 'gonadal vein draining into IVC'.

The renal vessels in the present study were described as unilateral or bilateral whether for arteries or veins. They were named double, triple, or quadruple according to the number and were named right or left according to the side. The renal vessels were named superior when cephalad to the renal vein (or renal artery) and inferior when caudad to them. They were also named hilar, superior polar, or inferior polar according to their attachment to the hilum, to the superior pole, or to the inferior pole, respectively. If a cadaver had more than 1 renal vein (or renal artery) on either side, then the course and attachment of that accessory renal vein (or artery) was studied in relation to the hilar renal vessels [13]. Other variations associated with the renal vessels in the region of the kidneys were recorded.

\section{Statistical analysis}

A two-sample $t$ test and Fisher exact tests were used to test for the association of renal vasculature variations with age at death, sex, and cause of death. Percentages and exact $95 \%$ confidence intervals (Cl) were used to estimate the incidence of aberrant and accessory renal vessels and associated renal vasculature variations. The McNemar test was used to test whether renal variations and accessory renal vessels were more commonly found on a specific side of the body. Fisher exact tests were also used to test for differences between males and females on the presence of accessory, hilar, and polar renal veins and arteries. SAS software version 9.4 (SAS Institute, Inc., Cary, NC) was used to conduct the statistical analyses. A $p<0.05$ was considered statistically significant.

\section{RESULTS}

During the Gross Anatomy course, 63 cadavers were dissected in the present study by first-year medical student volunteers as part of routine cadaveric dissection of the abdominal region resulting in 126 kidney specimens. Thirty-two (51\%) cadavers were males and 31 (49\%) were females. Age at the time of death ranged from 41 to 105 years with a mean (standard deviation [SD]) age of $75 \pm 13$ years. The most common causes of death were cardiac (54\%), pulmonary (46\%), vascular (29\%), and malignancy $(25 \%)$. Variations of the renal vessels were found in 7 cadavers (11\%; 95\% Cl 5-22\%) (Fig. 1). Some of the variations were associated with other anomalies like a polycystic kidney or variations in the common iliac or gonadal veins. The demographic characteristics of the 7 cadavers are presented in Table 1 .

\section{Anatomical descriptions}

Cadaver 460. It was found 3 RRVs that emerged from the kidney and crossed from the right side over the right psoas major muscle toward the IVC. A superior hilar RRV emerged straight from the hilum of the kidney, a second middle hilar RRV emerged from the lower half of the hilum of the kidney (which was abnormally extended) and was directed obliquely half an inch below the right hilar renal vein toward the IVC, and a third inferior polar RRV emerged from the inferior pole of the kidney and was directed toward the IVC. The right ureter appeared compressed between the middle hilar RRV superficially in its proximal part and by both the inferior polar RRV and the inferior polar right renal artery, near the inferior pole of the kidney, in its distal part (Fig. 2a). It had 2 renal arteries that emerged from the abdominal aorta and extended to the right side posterior to the IVC and over the right psoas major muscle toward the right kidney. A hilar right renal artery entered the extended hilum of the kidney posterior to the superior hilar RRV and posterior to the middle hilar RRV but anterior to the right ureter. An inferior polar right renal artery ran posterior to the right ureter toward the lower pole of the kidney after crossing anterior to the right gonadal vein. We also found a right gonadal vein with a high attachment to the IVC almost at the level of entry of the renal veins (Fig. 2a).

Cadaver 461. We found 4 RRVs: 1 hilar and 3 inferior polar RRVs emerged from the right kidney and crossed to the left side toward the IVC in close relation to each other (Fig. 2b). The hilar RRV emerged partially deep to the first inferior polar RRV and was directed horizontally to the left toward the IVC. The first inferior polar RRV, arising from the inferior pole of the right kidney in conjunction with the second and third inferior polar RRVs, ran obliquely upward crossing over the hilar RRV and over the hilar right renal artery, almost reaching the superior pole of the kidney. The second inferior polar RRV, emerging from 


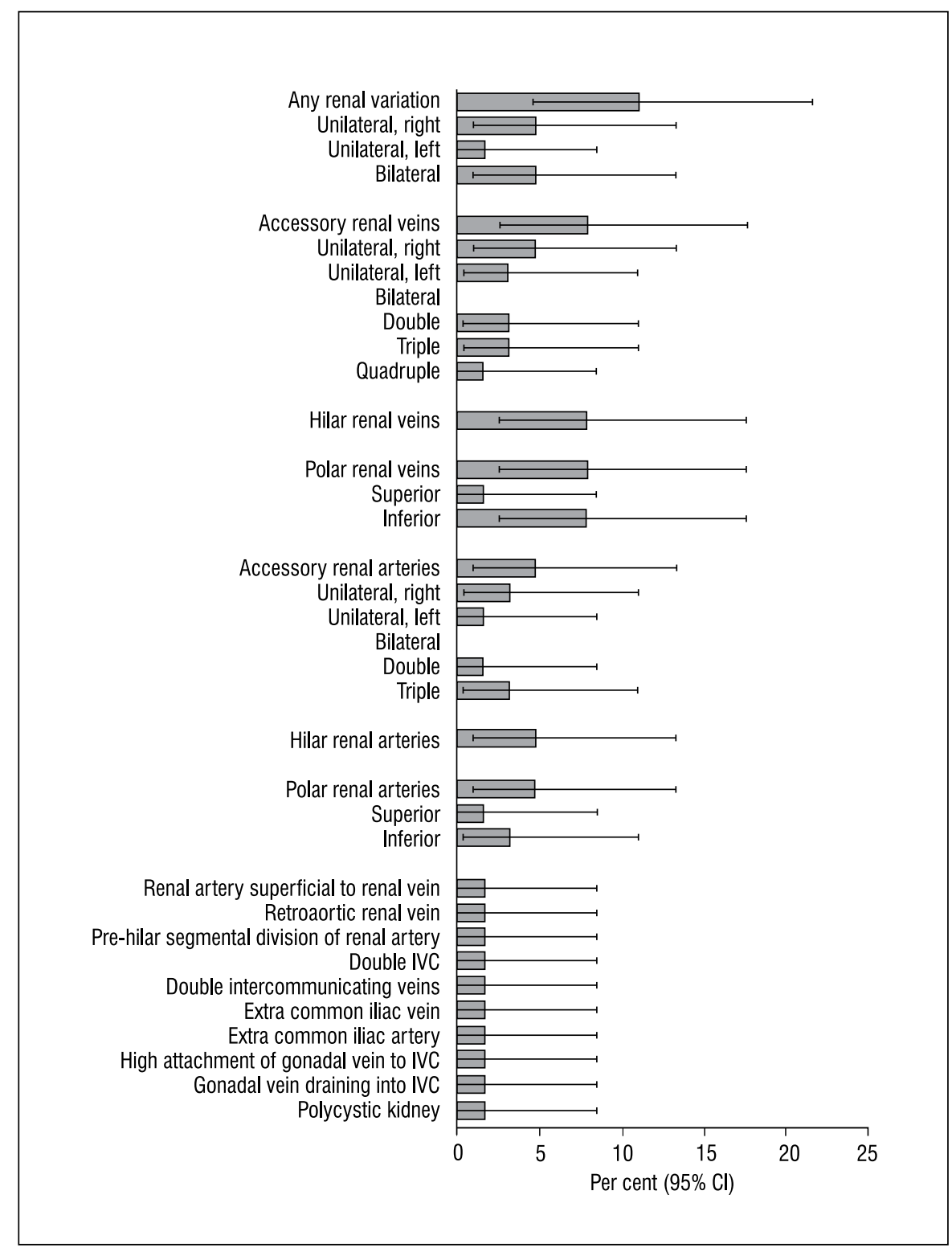

Figure 1. Incidence of variations in the renal vessels (per cent with 95\% confidence interval [CI]) in 63 cadavers; IVC — inferior vena cava.

Table 1. Demographic characteristics of the 7 cadavers with variations in the renal vasculature

\begin{tabular}{lccc}
\hline Cadaver & $\begin{array}{c}\text { Age at death } \\
\text { [years] }\end{array}$ & Sex & Cause of death \\
\hline 460 & 57 & Female & $\begin{array}{c}\text { Sudden cardiac death, atrial fibrillation, hypertensive heart disease, chronic kidney disease } \\
\text { Respiratory failure, pneumonia, metastatic lung cancer, renal failure } \\
\text { Ischaemic heart disease, severe atherosclerosis }\end{array}$ \\
513 & 73 & Female & Renal cancer with lung metastasis \\
522 & 91 & Female & Myocardial infarction \\
523 & 83 & Male & Male \\
529 & 49 & Female & Sudden death, coronary atherosclerosis, hyperlipidaemia, diabetes, \\
atrial fibrillation, hypertensive heart disease, renal failure \\
535
\end{tabular}



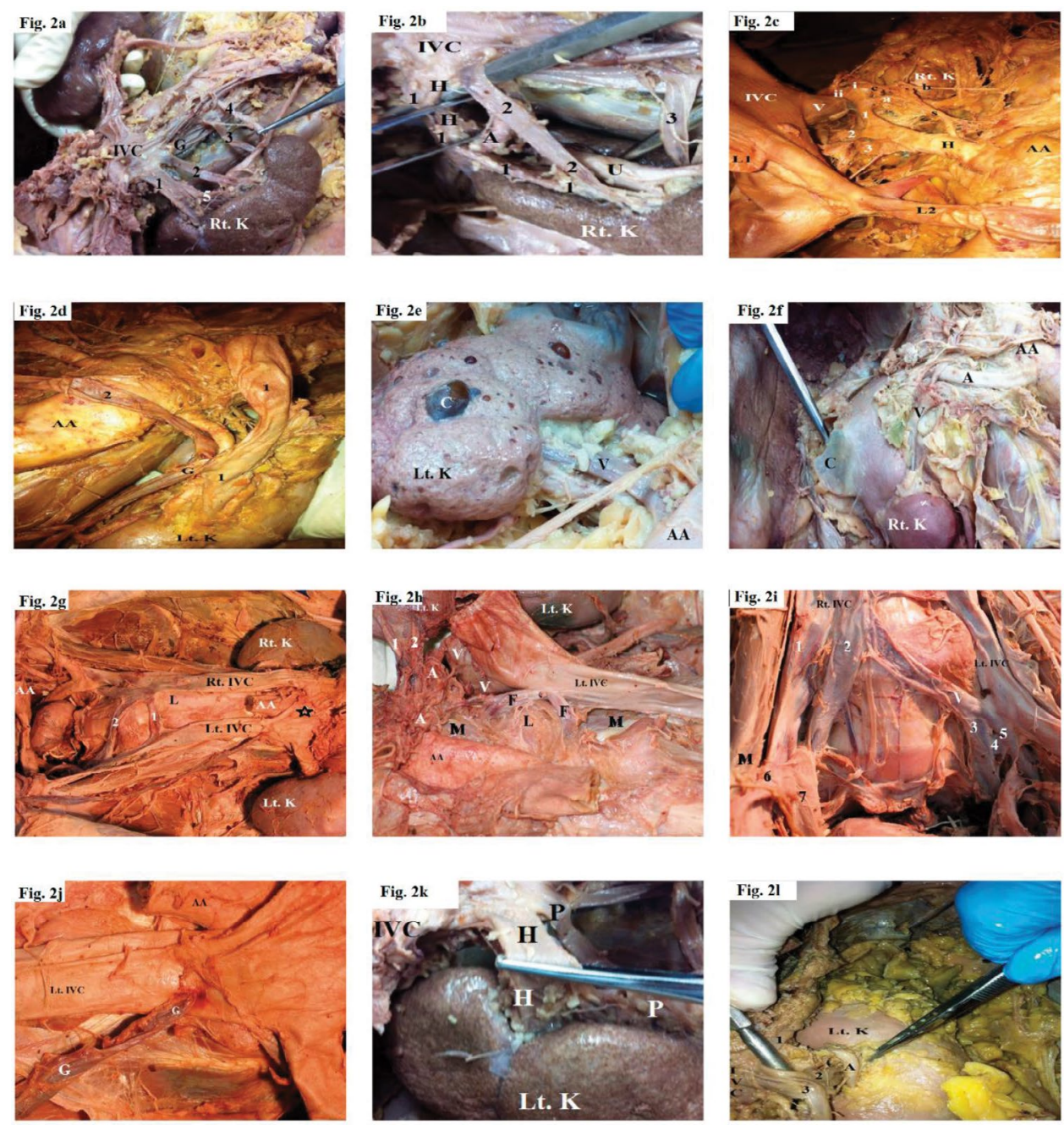

Figure 2. Dissected kidneys with variations in the renal vasculature; a (cadaver 460). Anterior view of the dissected right kidney showing 3 right renal veins: SHRRV (1), MHRRV (2), and IPRRV (3); 2 renal arteries: IPRRA (4) and HRRA (5); and RGV (G); b (cadaver 461). Anterior view of the dissected right kidney showing 4 renal veins: 1 HRRV $(H)$ and $3 \operatorname{IPRRV}(1,2$, and 3); c (cadaver 513). Anterior view of the dissected right kidney, IVC, and AA showing pre-hilar segmental division of the HRRA (H) into 3 segmental arteries; SPRRA (1), MPRRA (2), and IPRRA (3). SPRRA (1) had a suprarenal branch (a) and 2 other hilar branches (i and ii) curving around the HRRV (V). The HRRA (H) had a right RISA (S) that further subdivided into a suprarenal branch (b) and a RRCA (C). SHLRV (L1) and IHLRV (L2) were seen on the left side; $\mathbf{d}$ (cadaver 513). Showing SHLRV (L1) and IHLRV (L2). The SHLRV (L1) was cut to show the pre-hilar segmental division of the hilar right renal arteries into 3 polar right renal arteries. The IHLRV (L2) drained the LGV (G) as a normal tributary; e (cadaver 522). Anterior view of a dissected left kidney showing multiple cysts (C) with its LHRV (V) running deep to the AA; $f$ (cadaver 522). Anterior view of the dissected right kidney showing a single ruptured renal cyst (C); otherwise, the renal vasculature of the right kidney, including the RHRV (V) draining the kidney and the RHRA (A) branching of the AA, appeared normal; $\mathbf{g}$ (cadaver 523). Anterior view of the dissected right and left kidneys showing 2 IVC connected by an arch (star), AA (cut); lumbar vertebral bodies (L), superior intercommunicating veins (1), inferior intercommunicating veins (2); $\boldsymbol{h}$ (cadaver 523). A higher magnification of the proximal part of $\mathbf{g}$ showing the left IVC, AA, the lumbar vertebral bodies (L) a U-shaped sling of muscle fibres (F), the prevertebral muscles (M), HLRA (A), HLRV (V), which is cut, and 2 superior polar left renal arteries (1 and 2); $\mathbf{i}$ (cadaver 523). A higher magnification of the distal part of $\mathbf{g}$ showing the right and left IVC, 2 RCIV (1 and 2), inferior ICV (V), 3 LCIV (3, 4, and 5), 2 RCIA (6 and 7), and right psoas major muscle (M); $\mathbf{j}$ (cadaver 523). A higher magnification of the proximal part of $\mathbf{g}$ showing the left IVC and the LGV (G); $\mathbf{k}$ (cadaver 529). Anterior view of the dissected right kidney showing the HRRV emerging out of the hilum of the kidney toward the IVC and the IPRRV emerging out of the inferior pole of the kidney toward the IVC; I (cadaver 535). Anterior view of the dissected left kidney showing 3 renal veins emerging from the left kidney toward the IVC; the SPLRV emerging vertically downward from the superior pole of the kidney, the second MHLRV emerging horizontally from the hilum of the kidney, the third IPLRV emerging vertically upward from the inferior pole of the kidney, and the 3 veins merging toward the IVC. The HLRA entering the hilum of the kidney superficial to the MHLRV; AA — abdominal aorta; HLRA — hilar left renal artery; HLRV — hilar left renal vein; HRRA — hilar right renal artery; HRRV — hilar right renal vein; ICV — intercommunicating vein; IHLRV — inferior hilar left renal vein; IPLRV — inferior polar left renal vein; IPRRA — inferior polar right renal artery; IPRRV — inferior polar right renal vein; IVC — inferior vena cava; K — kidney; LCIV — left common iliac vein; LGV — left gonadal vein; LHRV — left hilar renal vein; Lt. — left; MHLRV — middle hilar left renal vein; MHRRV — middle hilar right renal vein; MPRRA — middle polar right renal artery; RCIA — right common iliac artery; RCIV — right common iliac vein; RGV — right gonadal vein; RHRA — right hilar renal artery; RHRV — right hilar renal vein; RISA — right inferior suprarenal artery; RRCA — right renal capsular artery; Rt. — right; SHLRV — superior hilar left renal vein; SHRRV — superior hilar right renal vein; SPLRA — superior polar left renal artery; SPLRV — superior polar left renal vein; SPRRA — superior polar right renal artery. 
the inferior pole of the kidney, ran obliquely upward where it entered the IVC very close to the hilar RRV. The third inferior polar RRV emerged from the inferior pole of the kidney and ran horizontally to the left where it entered the IVC (Fig. 2b).

Cadaver 513. We found pre-hilar segmental division of the right renal artery (Fig. 2c). It arose in a normal pattern from the abdominal aorta and ran horizontally and to the right behind the IVC. It then ran behind the hilar RRV and branched into the following: an inferior right suprarenal artery that subdivided into a suprarenal branch and a capsular branch to supply the right renal capsule, a superior polar renal artery that subdivided into a suprarenal branch to supply the suprarenal gland and 2 other branches that entered the kidney, and a middle polar right renal artery and inferior polar right renal artery that both entered the lower pole of the kidney (Fig. 2c). It also had 2 renal veins crossing from right to left transversely, bridging over the abdominal aorta and connecting the left kidney to the IVC. The superior hilar LRV which connected the IVC to the hilum of the left kidney was cut during dissection (Fig. 2c, d) to show the pre-hilar segmental division of the hilar right renal artery. The inferior hilar LRV connected the IVC to the lower pole of the kidney and drained the left gonadal vein as a normal tributary (Fig. 2d).

Cadaver 522. The hilar LRV reached the IVC deep to the abdominal aorta instead of superficial to it (i.e., retroaortic) (Fig. 2e). There were also multiple cysts in the left kidney (polycystic kidney). On the opposite side of the body, a single ruptured renal cyst was found on the outer convex border of the right kidney, and the rest of the surface of that kidney appeared normal (Fig. 2f).

Cadaver 523. We found the presence of 2 IVCs. One was normally present on the right side of the abdominal aorta, and the other was abnormally present to the left side of the abdominal aorta and connected by an arch that bridged over theabdominal aorta (Fig. 2g). The abdominal aorta was cut during dissection in its upper part (Fig. $2 \mathrm{~g}, \mathrm{~h}$ ) and reflected away from the lumbar vertebral bodies to show 2 superior and inferior intercommunicating veins connecting the 2 IVCs and bridging over the lumbar vertebral bodies (Fig. 2g). The left IVC (accessory IVC) was attached in its abnormal position to the left of the abdominal aorta and anterior to the lumbar vertebral bodies by a U-shaped sling of muscle fibres that were fused with the prevertebral muscles (Fig. 2h). The right IVC split distally into 2 right common iliac veins (Fig. 2i), and the left IVC combined with the lower intercommunicating vein that connected it to the right IVC and then split into 3 left common iliac veins (Fig. 2i). The left gonadal vein was seen as a tributary of the left IVC instead of being a tributary of the LRV (Fig. 2j). It had 2 right common iliac arteries of unknown origin but probably non-aortic origin; these 2 arteries appeared as a common short stem between the 2 right common iliac veins (Fig. 2i). The lateral right common iliac artery (right common iliac artery number 1), curved laterally to the right, superficial to the right common iliac vein (right common iliac vein number 1), and disappeared under the right psoas major muscle; the medial artery (right common iliac artery number 2) ran downward and medially toward the pelvis (Fig. 2i).

Cadaver $\mathbf{5 2 3}$ had 1 main hilar left renal artery and 2 superior polar left renal arteries. The hilar left renal artery ran to the left and entered the kidney deep to the hilar LRV, as normally expected, while the 2 superior polar left arteries entered the upper pole of the kidney (Fig. 2h).

Cadaver 529. It was found 2 RRVs. The hilar RRV emerged out of the hilum of the kidney and was directed straight to the left toward the IVC. An accessory inferior polar RRV emerged out of the inferior pole of the kidney and was directed obliquely upward and to the left toward the IVC (Fig. 2k).

Cadaver 535. We found 3 LRVs that emerged from the kidney toward the IVC. A superior polar LRV emerged vertically downward from the superior pole of the kidney, a second middle hilar LRV emerged horizontally from the kidney, and a third inferior polar LRV emerged vertically upward from the inferior pole of the kidney. The 3 LRVs merged toward the IVC (Fig. 2I). The same specimen also had the hilar left renal artery running from right to left to enter the kidney superficial (instead of deep) to the middle hilar LRV (Fig. 2l).

\section{Renal vasculature variation incidence and correlations}

There were no significant differences between those cadavers with or without variations in the renal vasculature and age at death (mean $\pm S D, 73 \pm 15$ years vs. $75 \pm 13$ years; $p=0.67)$ or $\operatorname{sex}(3 / 7[43 \%]$ males vs. 29/56 [52\%] females; $p=0.71$ ). Renal failure was the only cause of death that was significantly different between those with or without variations 


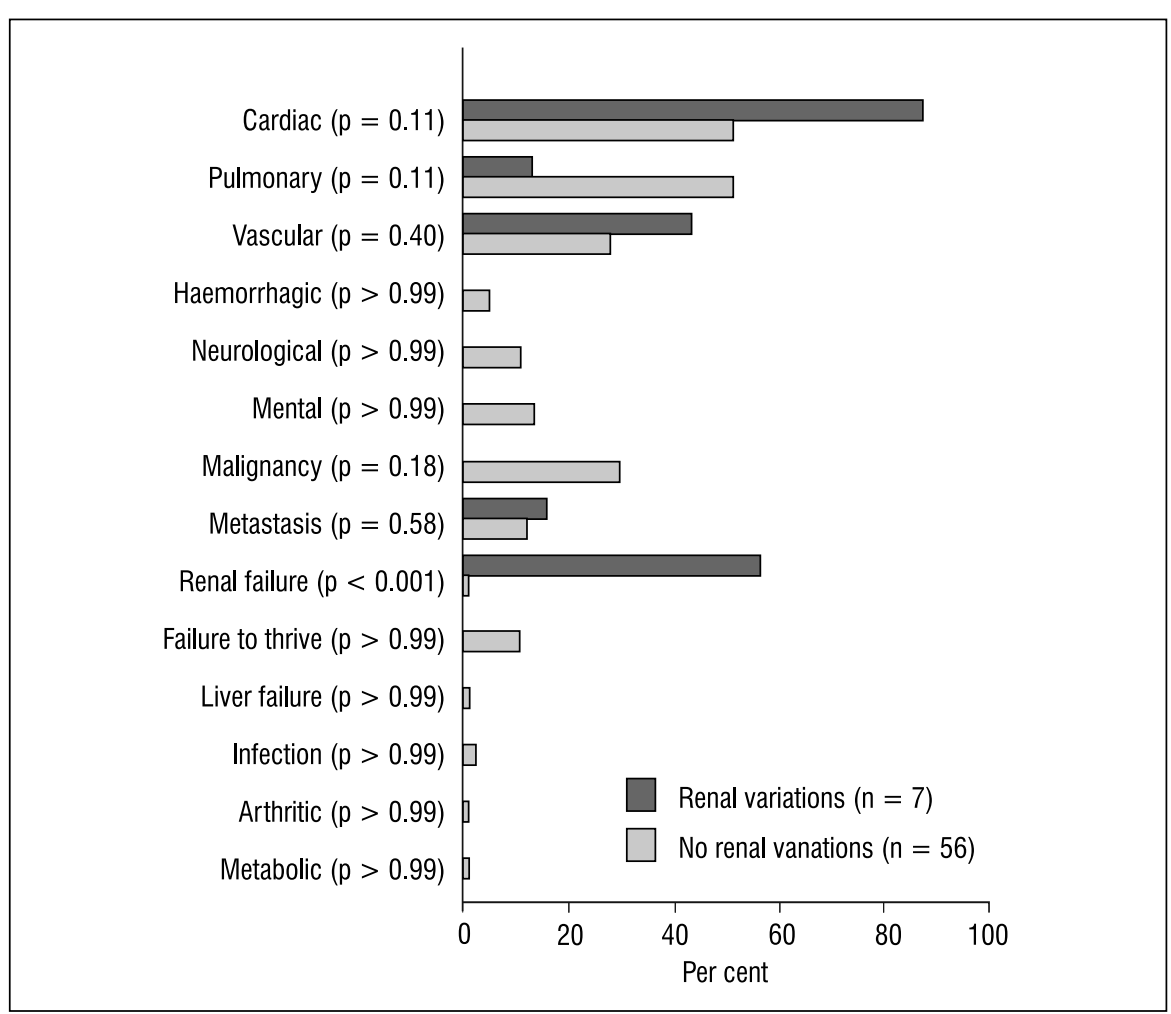

Figure 3. Comparisons of cause of death with presence or absence of renal variations.

in the renal vasculature $(4 / 7[57 \%]$ vs. $1 / 56[2 \%]$; $\mathrm{p}<0.001$ ) (Fig. 3).

The presence of accessory renal variations was unilateral on the right side in 3 cadavers (cadavers $460,461$, and 529; 5\%; $95 \% \mathrm{Cl} 1-13 \%)$, unilateral on the left side in 1 cadaver (cadaver 535; 2\%; $95 \%$ Cl 0-9\%), and bilateral in 3 cadavers (cadavers 513, 522 , and 523; 5\%; 95\% Cl 1-13\%) (Table 2). There was no significant difference between the right and left sides on the presence of renal vasculature variations (6/63 [10\%] vs. $4 / 63[6 \%] ; p=0.32$ ).

Accessory renal veins were found in 5 cadavers (cadavers 460, 461, 513, 529, and 535; 8\%; $95 \% \mathrm{Cl}$ 3-18\%) (Table 2). There was no significant difference between the right and left sides on the presence of accessory renal veins $(3 / 63[5 \%]$ vs. $2 / 63[3 \%] ; p=0.65)$. Double renal veins (1 accessory vein) were found in 2 cadavers (cadavers 513 and 529;3\%; 95\% Cl $0-11 \%$ ), triple renal veins ( 2 accessory veins) in 2 cadavers (cadavers 460 and $535 ; 3 \% ; 95 \% \mathrm{Cl}$ $0-11 \%$ ), and quadruple renal veins (3 accessory veins) in 1 cadaver (cadaver 461; 2\%; 95\% Cl 0-9\%) (Table 2).

The most common renal variations were the presence of hilar renal veins and polar renal veins, each occurring in 5 cadavers (cadavers 460, 461, 513, 529, and 535; 8\%; 95\% Cl 3-18\%) (Fig. 1, Table 2). Both superior and inferior polar renal veins were present in 1 cadaver (cadaver 535; $2 \% ; 95 \% \mathrm{Cl} 0-9 \%$ ), and inferior polar renal veins only were present in 4 cadavers (cadavers 460, 461, 513, and 529; 6\%; 95\% $\mathrm{Cl} 2-15 \%)$. There were no significant differences between males and females on the presence of accessory, hilar, or polar renal veins (all $p=0.20$ ) (Table 2 ).

Accessory renal arteries were found in 3 cadavers (cadavers 460, 513, and 523; 5\%; 95\% Cl 1-13\%) (Table 2). There was no significant difference between the right and left sides on the presence of accessory renal arteries $(2 / 63[3 \%]$ vs. $1 / 63[2 \%] ; p=0.56)$. Double renal arteries ( 1 accessory artery) were found in 1 cadaver (cadaver 460; $2 \% ; 95 \% \mathrm{Cl} 0-9 \%$ ), and triple renal arteries (2 accessory arteries) were found in 2 cadavers (cadavers 513 and 523; 3\%; 95\% Cl 0-11\%) (Table 2).

Hilar renal arteries were present in 3 cadavers (cadavers 460, 513, and 523; 5\%; 95\% Cl 1-13\%), and polar renal arteries were present in 3 cadavers (cadavers 460,513 , and 523; 5\%; $95 \% \mathrm{Cl} 1-13 \%$ ), including superior polar renal arteries only in $1 \mathrm{ca}-$ daver (cadaver 523; 2\%; 95\% Cl 0-9\%) and inferior polar renal arteries only in 2 cadavers (cadavers 460 
Table 2. Relationship of sex with variations in renal vasculature in 63 dissected cadavers

\begin{tabular}{|c|c|c|c|c|}
\hline Type of variation & $\begin{array}{c}\text { All }(\mathbf{n}=63) \\
\mathrm{n}(\%) ; 95 \% \text { CI }\end{array}$ & $\begin{array}{c}\text { Male }(n=32) \\
n(\%)^{\mathrm{a}}\end{array}$ & $\begin{array}{c}\text { Female }(n=31) \\
n(\%)^{\mathrm{a}}\end{array}$ & $\mathbf{P b}$ \\
\hline Any renal variation & $7(11 \%) ; 5-22$ & $3(10 \%)$ & $4(13 \%)$ & 0.71 \\
\hline Unilateral, right & $3(5 \%) ; 1-13$ & $0(0 \%)$ & $\begin{array}{c}3(10 \%) \\
\text { Cadavers } 460,461, \\
\text { and } 529\end{array}$ & \\
\hline Unilateral, left & $1(2 \%) ; 0-9$ & $\begin{array}{c}1(3 \%) \\
\text { Cadaver } 535\end{array}$ & $0(0 \%)$ & \\
\hline Bilateral & $3(5 \%) ; 1-13$ & $\begin{array}{c}2(6 \%) \\
\text { Cadavers } 522 \text { and } 523\end{array}$ & $\begin{array}{c}1(3 \%) \\
\text { Cadaver } 513\end{array}$ & \\
\hline Accessory renal veins & $5(8 \%) ; 3-18$ & $1(3 \%)$ & $4(13 \%)$ & 0.20 \\
\hline Unilateral, right & $3(5 \%) ; 1-13$ & $0(0 \%)$ & $3(10 \%)$ & \\
\hline Unilateral, left & $2(3 \%) ; 0-11$ & $1(3 \%)$ & $1(3 \%)$ & \\
\hline Bilateral & $0(0 \%) ; 0-9$ & $0(0 \%)$ & $0(0 \%)$ & \\
\hline Double renal veins ${ }^{\mathrm{C}}$ & $2(3 \%) ; 0-11$ & $0(0 \%)$ & $\begin{array}{c}2(6 \%) \\
\text { Cadavers } 513(\mathrm{~L}) \\
\text { and } 529(\mathrm{R})\end{array}$ & \\
\hline Triple renal veins ${ }^{c}$ & $2(3 \%) ; 0-11$ & $\begin{array}{c}1(3 \%) \\
\text { Cadaver } 535 \text { (L) }\end{array}$ & $\begin{array}{c}1(3 \%) \\
\text { Cadaver } 460(\mathrm{R})\end{array}$ & \\
\hline Quadruple renal veins ${ }^{c}$ & $1(2 \%) ; 0-9$ & $0(0 \%)$ & $\begin{array}{c}1(3 \%) \\
\text { Cadaver } 461(\mathrm{R})\end{array}$ & \\
\hline Hilar renal veins & $5(8 \%) ; 3-18$ & $\begin{array}{c}1(3 \%) \\
\text { Cadaver 535 (L) }\end{array}$ & $\begin{array}{c}4(13 \%) \\
\text { Cadavers } 460(\mathrm{R}), \\
461(\mathrm{R}), 513(\mathrm{R}) \\
\text { and } 529(\mathrm{R})\end{array}$ & 0.20 \\
\hline Polar renal veins & $5(8 \%) ; 3-18$ & $1(3 \%)$ & $4(13 \%)$ & 0.20 \\
\hline Superior polar renal veins & $1(2 \%) ; 0-9$ & $\begin{array}{c}1(3 \%) \\
\text { Cadaver } 535 \text { (L) }\end{array}$ & $0(0 \%)$ & \\
\hline Inferior polar renal veins & $5(3 \%) ; 3-18$ & $\begin{array}{c}1(3 \%) \\
\text { Cadaver } 535 \text { (L) }\end{array}$ & $\begin{array}{c}4(13 \%) \\
\text { Cadavers } 460(\mathrm{R}), \\
461(\mathrm{R}), 513(\mathrm{~L}) \\
\text { and } 529(\mathrm{R})\end{array}$ & \\
\hline Accessory renal arteries & $3(5 \%) ; 1-13$ & $1(3 \%)$ & $2(6 \%)$ & 0.61 \\
\hline Unilateral, right & $2(3 \%) ; 0-11$ & $0(0 \%)$ & $2(6 \%)$ & \\
\hline Unilateral, left & $1(2 \%) ; 0-9$ & $1(3 \%)$ & $0(0 \%)$ & \\
\hline Bilateral & $0(0 \%) ; 0-9$ & $0(0 \%)$ & $0(0 \%)$ & \\
\hline Double renal arteries ${ }^{C}$ & $1(2 \%) ; 0-9$ & $0(0 \%)$ & $\begin{array}{c}1(3 \%) \\
\text { Cadaver } 460 \text { (R) }\end{array}$ & \\
\hline Triple renal arteries ${ }^{C}$ & $2(3 \%) ; 0-11$ & $\begin{array}{c}1(3 \%) \\
\text { Cadaver } 523(\mathrm{~L})\end{array}$ & $\begin{array}{c}1(3 \%) \\
\text { Cadaver } 513(\mathrm{R})\end{array}$ & \\
\hline Hilar renal arteries & $3(5 \%) ; 1-13$ & $\begin{array}{c}1(3 \%) \\
\text { Cadaver } 523 \text { (L) }\end{array}$ & $\begin{array}{c}2(6 \%) \\
\text { Cadavers } 460(\mathrm{R}) \\
\text { and } 513(\mathrm{R} \%)\end{array}$ & 0.61 \\
\hline Polar renal arteries & $3(5 \%) ; 1-13$ & $1(3 \%)$ & $2(6 \%)$ & 0.61 \\
\hline Superior polar renal arteries & $1(2 \%) ; 0-9$ & $\begin{array}{c}1(3 \%) \\
\text { Cadaver } 523 \text { (L) }\end{array}$ & $0(0 \%)$ & \\
\hline Inferior polar renal arteries & $2(3 \%) ; 0-11$ & $0(0 \%)$ & $\begin{array}{l}2(6 \%) \\
\text { Cadavers } 460(\mathrm{R}) \\
\text { and } 513(\mathrm{R})\end{array}$ & \\
\hline Renal artery superficial to renal vein & $1(2 \%) ; 0-9$ & $\begin{array}{c}1(3 \%) \\
\text { Cadaver } 535 \text { (L) }\end{array}$ & $0(0 \%)$ & $>0.99$ \\
\hline
\end{tabular}


Table 2. cont. Relationship of sex with variations in renal vasculature in 63 dissected cadavers

\begin{tabular}{|c|c|c|c|c|}
\hline Type of variation & $\begin{array}{c}\text { All (n= 63) } \\
\mathrm{n}(\%) ; 95 \% \text { CI }\end{array}$ & $\begin{array}{c}\text { Male }(\mathbf{n}=32) \\
n(\%)^{\mathrm{a}}\end{array}$ & $\begin{array}{c}\text { Female }(\mathbf{n}=31) \\
n(\%)^{\mathrm{a}}\end{array}$ & $\mathbf{P b}$ \\
\hline Retroaortic renal vein & $1(2 \%) ; 0-9$ & $\begin{array}{c}1(3 \%) \\
\text { Cadaver 522 (L) }\end{array}$ & $0(0 \%)$ & $>0.99$ \\
\hline Pre-hilar segmental division of renal artery & $1(2 \%) ; 0-9$ & $0(0 \%)$ & $\begin{array}{c}1(3 \%) \\
\text { Cadaver } 513(\mathrm{R})\end{array}$ & 0.49 \\
\hline Double IVC & $1(2 \%) ; 0-9$ & $\begin{array}{c}1(3 \%) \\
\text { Cadaver } 523 \text { (L) }\end{array}$ & $0(0 \%)$ & $>0.99$ \\
\hline Double intercommunicating veins between 2 IVC & $1(2 \%) ; 0-9$ & $\begin{array}{c}1(3 \%) \\
\text { Cadaver } 523 \text { (L) }\end{array}$ & $0(0 \%)$ & $>0.99$ \\
\hline Extra common iliac vein & $1(2 \%) ; 0-9$ & $\begin{array}{c}1(3 \%) \\
\text { Cadaver } 523 \text { (B) }\end{array}$ & $0(0 \%)$ & $>0.99$ \\
\hline Extra common iliac artery & $1(2 \%) ; 0-9$ & $\begin{array}{c}1(3 \%) \\
\text { Cadaver } 523(\mathrm{R})\end{array}$ & $0(0 \%)$ & $>0.99$ \\
\hline High attachment of gonadal vein to IVC & $1(2 \%) ; 0-9$ & $0(0 \%)$ & $\begin{array}{c}1(3 \%) \\
\text { Cadaver } 460 \text { (R) }\end{array}$ & 0.49 \\
\hline Gonadal vein draining into IVC & $1(2 \%) ; 0-9$ & $\begin{array}{c}1(3 \%) \\
\text { Cadaver } 523 \text { (L) }\end{array}$ & $0(0 \%)$ & $>0.99$ \\
\hline Polycystic kidney & $1(2 \%) ; 0-9$ & $\begin{array}{c}1(3 \%) \\
\text { Cadaver } 522(\mathrm{~B})\end{array}$ & $0(0 \%)$ & $>0.99$ \\
\hline
\end{tabular}

${ }^{a}$ Cadavers having a specific renal vasculature variation and the side of the variation are listed when appropriate.

bP values from Fisher exact test comparing males and females on types of renal variations.

'Double renal veins and arteries had 1 accessory vein or artery, triple renal veins and arteries had 2 accessory veins or arteries, and quadruple renal veins had 3 accessory veins.

$\mathrm{B}$ - bilateral variation; $\mathrm{Cl}$ — confidence interval; IVC — inferior vena cava; $\mathrm{L}$ — left side variation; $\mathrm{R}$ — right side variation

and $513 ; 3 \% ; 95 \% \mathrm{Cl} 0-11 \%)$. There were no significant differences between males and females on the presence of accessory, hilar, or polar renal arteries (all $\mathrm{p}=0.61$ ) (Table 2).

Of the 63 cadavers, the renal artery superficial to the renal vein, the retroaortic renal vein, and prehilar segmental division of the renal artery were each present in 1 cadaver (cadaver 535, cadaver 522, and cadaver 513, respectively; 2\%; 95\% Cl 0-9\%) (Table 2). Double IVC, double intercommunicating veins between 2 IVC, extra common iliac veins, and an extra common iliac artery were all present in 1 cadaver (cadaver 523; 2\%; 95\% Cl 0-9\%) (Fig. 1, Table 2). Abnormal gonadal veins were present in 2 cadavers (cadavers 460 and 523; 3\%; 95\% Cl 0-11\%), including high attachment of a gonadal vein to the IVC in 1 cadaver (cadaver 460; $2 \% ; 95 \% \mathrm{Cl} 0-9 \%$ ) and a left gonadal vein draining into the IVC in the other cadaver (cadaver 523; 2\%; 95\% Cl 0-9\%). Polycystic kidneys were found bilaterally in 1 cadaver (cadaver 522; 2\%; 95\% Cl 0-9\%).

\section{DISCUSSION}

A combination of patterns of accessory renal veins and arteries were seen in the present study. Variations in the renal vasculature were present in $11 \%$ of cadavers. The renal variations appeared to be unilateral on the right side in $5 \%$ of cadavers, unilateral on the left side in $2 \%$ of cadavers ( 1 cadaver), and bilateral in $5 \%$ of cadavers. We found no significant difference between the right and left sides of the body and the presence of renal variations. These results are different from those of Kaneko et al. [13], who reported similar variations in the renal vasculature in $26 \%$ of cadavers. This difference in incidence of variations may be explained by sample size: in the present study, we examined 63 cadavers, and the study by Kaneko et al. [13] examined 190 cadavers. However, in contrast to the present study, no correlation between venous and arterial variations in the renal vasculature was described by Kaneko et al. [13].

Several classifications have been made regarding the drainage patterns of the renal veins. Smithuis [28] reported only 2 primary tributaries (upper and lower), and Merklin and Mitchels [15] recognised the posterior primary tributary of the renal vein and emphasized its clinical importance its retropelvic position. Gillot [9] also recognised the posterior primary tributary of the renal vein; however, he did not include it in his classification. Sampaio and Aragão [23] reported 
3 primary tributaries (upper, middle, and lower) and described 6 variations for these renal veins while Chaung et al. [6] proposed a different classification for the LRV only (retroaortic, preaortic, circumaortic, and supernumerary). In a cadaveric study, Yi et al. [34] had a similar classification to Chaung et al. [6] where they described the 3 most common variations in the LRV (circumaortic, retroaortic, and retropelvic). In the present study, we found only the retroaortic type of variation in the LRV in 1 cadaver.

In the present study, we classified the renal veins according to their number, origin, and destination into two types: accessory hilar renal vein and accessory polar renal veins where the accessory hilar renal vein was additional to the usual primary (lobar) tributaries of the hilar renal vein and the accessory polar renal vein was defined as one that was draining separately from either pole of the kidney independently into the IVC, away from the hilar renal vein.

This classification differs from Satyapal's classification [24], where he described three types of renal venous drainage patterns (types IA, IB, IIA, IIB, and III) depending on the number of primary tributaries of the renal vein, the presence or absence of a posterior primary tributary, and the presence of an additional renal vein. The accessory polar renal vein described in the present study corresponds to type III described by Satyapal [24].

The classification in the present study was also different from the classification described by Zhu et al. [35] in a retrospective computed tomography (MDCT) study on 1452 patients who underwent MDCT angiography. They classified the variations in the LRVs according to the origin, destination, number, branches, length, and course into five types from I to $\checkmark$ resulting in the following classifications: circumaortic $(2.1 \%)$, retroaortic $(2.1 \%)$, abnormal reflux $(1.7 \%)$, late venous confluence $(0.9 \%)$, and a fifth rare type $(0.3 \%)$. Zhu et al. [35] also classified the variations in the RRVs into three types from I to III resulting in the following classifications: additional (18.7\%), abnormal reflux $(0.4 \%)$, and a third rare type $(0.1 \%)$.

The most common renal variations in the present study were the presence of accessory hilar renal veins and accessory polar renal veins. Accessory hilar renal veins were found in $8 \%$ of cadavers, and accessory polar renal veins were also found in $8 \%$ of cadavers. Of these, $3 \%$ of cadavers had 1 accessory renal vein, $3 \%$ had 2 accessory renal veins, and 2\% (1 cadaver) had 3 accessory renal veins. These findings contradict those of Kaneko et al. [13], who reported unilateral accessory renal veins in $13 \%$ of cadavers and bilateral accessory renal veins in only $1 \%$ ( 1 cadaver).

The published literature includes unexplained discrepancies between findings of renal vasculature variations. Previous studies $[12,18]$ found variations in renal veins were more common on the right side than the left side. Bergman et al. [4] also suggested that these variations were rare on the left side $(1 \%)$ compared with the right side (28\%). However, Baptista-Silva et al. [2] reported a $92 \%$ prevalence of variations in the renal veins on the left side and an $8 \%$ prevalence of variations on the right side in a study of 342 living donors of nephrectomy. Recently, Zhu et al. [35] reported a $19 \%$ prevalence of variations in the renal veins on the right side, a $7 \%$ prevalence of variations on the right side, and $1.7 \%$ on both sides in a retrospective MDCT study. In the present study, single or double accessory renal veins were present either on the right or left side of the body, but accessory triple renal veins were present only on the right side. Further, neither the right nor the left side was more likely to have accessory renal veins. These discrepancies in the incidence of variations in renal vasculature may be attributed to differences in the number of participants in each study or to the different methods of the studies. MDCT has been proven to be a very sensitive, specific, and accurate method for evaluation of renal vein variations compared with many other methods because of its high resolution [35]. However, this finding contradicts $\mathrm{Yi}$ et al. [34] who reported that it was much easier to detect circumaortic variation of the LRV via cadaveric dissection compared with MDCT or angiography.

Accessory renal arteries originating from the abdominal aorta were observed in $5 \%$ of cadavers in the present study. Ozkan et al. [20], Kaneko et al. [13] and Saldarriaga et al. [22] reported about 25\% of patients had accessory renal arteries, and Vatsala et al. [33] observed them in $50 \%$ of cadavers. In the present study, only $2 \%$ of cadavers had only 1 accessory renal artery, which contradicts the findings of Vatsala et al. [33], who found $22 \%$ of kidneys had only 1 accessory renal artery. It also contradicts Ozkan et al. [20], who reported a single accessory renal artery in $76 \%$ of kidneys. In the present study, $3 \%$ of cadavers had 2 accessory renal arteries, which supports the findings of Vatsala et al. [33], who also found 3\% of kidneys had 2 accessory renal arteries, but contradicts Ozkan et al. [20], who reported multiple accessory 
renal arteries in $24 \%$ of kidneys. These discrepancies in findings of renal vasculature variations between the present study and previous studies $[13,22,33]$ may be attributed to different study populations. Kaneko et al. [13] and Saldarriaga et al. [22] studied patients undergoing surgical or radiological procedures. In the present study and in Vatsala et al. [33], cadavers were studied for renal vasculature variations. Further, the difficulty or ease in separating the renal vasculature and their surrounding structures and the resilience or compliance of the separated tissues may differ between cadavers and living patients.

In the present study, accessory renal arteries were only found unilaterally on the right side of the body in $5 \%$ of cadavers. Ozkan et al. [20] reported unilateral accessory renal arteries on the right side of the body in $16 \%$ of kidneys, unilateral accessory renal arteries on the left side in $13 \%$ of kidneys, and bilateral accessory renal arteries in 5\% of kidneys. These data by Ozkan et al. [20] were similar to Petru et al. [21], who reported unilateral accessory renal arteries on the right side of the body in $2 \%$ of kidneys, unilateral accessory renal arteries on the left side in 15\% of kidneys, and bilateral accessory renal arteries in $2 \%$ of kidneys. Vatsala et al. [33] reported $18 \%$ of kidneys had unilateral accessory renal arteries, and $8 \%$ of kidneys had bilateral ones. Kaneko et al. [13] found unilateral accessory renal arteries in $21 \%$ of patients and bilateral accessory renal arteries in only $5 \%$. These variations in the incidence of the side of the renal vasculature variation may be attributed to differences in the number of cadavers or patients in each study, which varied from 20 to 190 . Differences may also be related to the age or sex of the cadavers or patients. Most of these previous studies did not report age or sex information [13, 33]. As a result, we could not make comparisons with our data.

Few studies have investigated the relationship between age or sex and the incidence of renal vascular variations. In the present study, there was no significant difference in age at death between those with or without renal variations. This finding was in agreement with a study by Harrison et al. [11], who also reported no significant differences for their age and sex study groups and the incidence of renal vascular variations, and with a recent MDCT study by Zhu et al. [35], who found no statistically significant correlation in the incidence of variations between the right and LRVs and gender. There were no significant differences between males and females for the pres- ence of renal variations in the present study, which is consistent with the findings of Zhu et al. [35]. However, Harrison et al. [11] did report a minor increase in the incidence of renal variations in females. In another study, Satyapal et al. [25] reported a male predominance of $28 \%$ versus only $5.1 \%$ for females for the incidence of accessory renal arteries. Cicekcibasi et al. [7] found a similar male predominance of $25 \%$ for the incidence of renal variations among human foetuses with the preference for male predominance on the right side of the body. To our knowledge, few studies have retrospectively correlated the cause of death with the incidence of renal vascular variations $[11,25]$, and studies that described the variations in the renal vasculature patterns were mostly case studies $[8,10,18]$. In the present study, renal failure was the only cause of death that was significantly different between those with or without renal variations.

In the present study, accessory renal arteries arising from the abdominal aorta appeared to be attached to the hila of kidneys in $5 \%$ of cadavers and attached to the poles in another $5 \%$ of cadavers. In a study by Petru et al. [21], of the 54 kidneys that had accessory renal arteries, 52\% entered the kidneys through their hila, $9 \%$ entered through their superior pole, and $30 \%$ entered through their inferior pole; all these arteries arose from the abdominal aorta. These findings were similar to those of Budhiraja et al. [5], who reported that accessory superior polar renal arteries emerged from the abdominal aorta in $11 \%$ of cases. Awareness of this unusual renal vascular pattern may be helpful for surgeons performing vascular reconstruction of congenital renal anomalies [10] and endoscopic renal surgeries or treating renal arterial stenosis [18].

In the present study, pre-hilar segmentation of the renal artery occurred in 1 cadaver. The main trunk of the right renal artery had a right suprarenal artery and supplied the kidney by 1 hilar and 2 polar arteries. The superior polar renal artery had a branch to the suprarenal gland and then divided into 2 other branches that entered the hilum of the right kidney. A similar finding was described by Tanyeli et al. [31] who added that a main trunk of the right renal artery ramified into the suprarenal gland while supplying the kidney with 2 hilar arteries. Nayak et al. [19] also described pre-hilar segmental division in the renal artery and vein with a left gonadal vein crossing in front of the left renal vessels in a tortuous course. Because safe operative exposure of the kidneys and clamping of the renal arteries to control bleeding during man- 
agement of trauma to the kidneys is mandatory [13], variations in the incidence of pre-hilar segmentation of the renal arteries may make it difficult to identify the renal arteries and could jeopardize the process of bleeding control.

In the present study, the hilar left renal artery was superficial to the accessory middle LRV instead of being deep to it (i.e., retroaortic renal vein) in 1 cadaver (2\%). Petru et al. [21] also found $2 \%$ of kidneys had retroaortic renal veins where renal arteries entered the kidney deep to the ureter instead of superficial to it. This reversal in the arrangement of the renal artery and the renal vein may be problematic during retraction of the renal vein prior to renal transplantation or renal artery embolisation. Ligation of the wrong renal vessel could result in the degeneration of a certain segment of the kidney since the renal arteries are considered functional end arteries [33].

The presence of these accessory renal arteries in such varying patterns may result from a complication in the process of foetal development. As a result, these arteries retain their embryonic origin from the lateral splanchnic vessels [29]. Normally, the process of foetal development starts in the pelvis where the kidneys acquire their blood supply from the common iliac arteries [17, 29] or from the superior mesenteric or coeliac arteries [29]. After ascending to the lumbar region, the kidneys change the source of blood supply to the aorta [17]. However, Ozkan et al. [20] suggested this variation in the renal artery resulted from a failure of complete degeneration of the embryonic mesonephric arteries supplying the kidneys, gonads, and suprarenal glands, leaving more than 1 mesonephric artery.

In the present study, accessory renal veins all drained into the IVC. In a study by Malcic-Gürbüz et al. [14], a branching LRV was described where the upper branch drained into the azygos vein and the lower 2 branches drained into the IVC. In the present study, 2 LRVs were seen crossing from right to left transversely, bridging over the abdominal aorta and connecting the left kidney to the IVC. Previous studies [2], Senecail et al. [27] described a venous ring encircling the abdominal aorta. Both types of variations in the renal veins may result from the persistence of the renal venous collar, which normally disappears during the development of the embryo.

The persistence of the renal venous collar may also explain other results of the present study, such as the presence of double IVC connected by double intercommunicating veins, the presence of an extra left common iliac vein as an extra tributary to the right IVC, and the left gonadal vein draining into the left IVC instead of draining into the LRV. Asala et al. [1] and Nayak et al. [18] reported a right gonadal vein draining into the renal vein on the right side of the body instead of draining into the IVC. The presence of these accessory renal veins with such varying patterns may be because of an error in merging of the temporary branches of the supracardinal, subcardinal, and postcardinal veins that form the IVC. As a result, these temporary veins have numerous anastomoses between them which result in the formation of the renal venous collar described above.

In the present study, 3 renal veins (2 hilar and 1 accessory inferior polar) emerged from the right kidney and crossed toward the IVC, compressing the right ureter. This finding is similar to that of Mishra et al. [16] who described a compressed ureter between the accessory renal artery and the accessory inferior polar renal vein, anteriorly, and the hilar renal vein posteriorly. They added that this kind of compression may cause hydronephrosis. Uzmansel et al. [32] also suggested that the presence of accessory renal vessels may lead to several clinical complications, one of which was hydronephrosis. This compression proposed by Mishra et al. [16] may explain the cystic kidney of the present study, which had a LRV reaching the IVC deep to the abdominal aorta. This explanation is supported by Bayramoglu et al. [3], who suggested that the presence of accessory renal vessels posterior to the ureter with a location close to the ureteropelvic junction may be a predisposing factor for the occurrence of hydronephrosis and cystic kidneys.

In the present study, variations in the renal venous pattern were more common than variations in the renal arterial pattern: accessory renal veins were found in $8 \%$ of cadavers, but accessory renal arteries were only found in $5 \%$ of cadavers. This result is in agreement with $\mathrm{Yi}$ et al. [34], who found that variations in the renal veins was the most common type of renal vasculature variation in Japanese cadavers. However, this result contradicts other studies $[4,10,26]$ that reported renal arterial variations were more common than renal venous variations. These contradictory findings suggest that the geographical origins of the cadavers are not a key factor in explaining differences in the incidence of variations of renal vasculature. 


\section{CONCLUSIONS}

The present study provided new data about variations in the renal vasculature and other vessels around the kidneys that may elucidate the operative anatomy of this region. Greater awareness of these variations may be particularly useful for surgeons and radiologists concerned with this anatomical region. Because of the relatively small number of cadavers examined, the present study may have not detected some rare variations seen in other larger studies, so future studies with larger sample sizes are recommended to replicate the findings of the present study. Because we used formaldehyde-fixed cadavers in the present study, which have less mobility and flexibility of the tissues, future studies using angiography of the renal vessels are recommended to better provide more meaningful data.

\section{Acknowledgements}

We thank the 63 persons who donated their cadavers, making this study possible, and we thank the 10 student volunteers who helped in the dissection of the renal vessels and the collection of the data. We also thank Deborah Goggin, MA, ELS, for her editorial assistance.

\section{REFERENCES}

1. Asala S, Chaudhary SC, Masumbuko-Kahamba N, et al. Anatomical variations in the human testicular blood vessels. Ann Anat. 2001; 183(6): 545-549, doi: 10.1016/ S0940-9602(01)80064-9, indexed in Pubmed: 11766526.

2. Baptista-Silva J, Veríssimo M, Castro M, et al. Anatomical study of the renal veins observed during 342 living-donor nephrectomies. Sao Paulo Medical Journal. 1997; 115(3): 1456-1459, doi: 10.1590/s1516-31801997000300011.

3. Bayramoglu A, Demiryurek D, Erbil KM. Bilateral additional renal arteries and an additional right renal vein associated with unrotated kidneys. Saudi Med J. 2003; 24(5): 535-537, indexed in Pubmed: 12847633.

4. Bergman RA, Thompson SA, Afifi AK, Saadeh FA. ompendium of Human Anatomic Variation. Urban \& Schwarzenberg, Baltimore 1988: Baltimore.

5. Budhiraja V, Rastogi R, Asthana AK. Variant origin of superior polar artery and unusual hilar branching pattern of renal artery with clinical correlation. Folia Morphol. 2011; 70(1): 24-28, indexed in Pubmed: 21604249.

6. Chuang VP, Mena CE, Hoskins PA, et al. Congenital anomalies of the inferior vena cava. Review of embryogenesis and presentation of a simplified classification. Br J Radiol. 1974; 47(556): 206-213, doi: 10.1259/0007-1285-47-556206, indexed in Pubmed: 4824552.

7. Ciçekcibaşi AE, Ziylan T, Salbacak A, et al. An investigation of the origin, location and variations of the renal arteries in human fetuses and their clinical relevance. Ann Anat.
2005; 187(4): 421-427, doi: 10.1016/j.aanat.2005.04.011, indexed in Pubmed: 16163857.

8. Fernandes R, Conte F, Favorito LA, et al. Triple Right Renal Vein: An Uncommon Variation. Int J Morphol. 2005; 23(3): 231-233, doi: 10.4067/s0717-95022005000300007.

9. Gillot $C$. The left renal vein: anatomical study, angiographic aspects and surgical approach. Anat Clin. 1978; 1: 135-156.

10. Gupta V, Kotgirwar S, Trivedi R, et al. Bilateral variations in renal vasculature. Int J Anat Var. 2010; 3: 53-55.

11. Harrison LH, Flye MW, Seigler HF. Incidence of anatomical variants in renal vasculature in the presence of normal renal function. Ann Surg. 1978; 188(1): 83-89, indexed in Pubmed: 352280.

12. Janschek ECS, Rothe AU, Hölzenbein TJ, et al. Anatomic basis of right renal vein extension for cadaveric kidney transplantation. Urology. 2004; 63(4): 660-664, doi: 10.1016/j. urology.2003.11.010, indexed in Pubmed: 15072874.

13. Kaneko N, Kobayashi Y, Okada Y, et al. Anatomic variations of the renal vessels pertinent to transperitoneal vascular control in the management of trauma. Surgery. 2008; 143(5): 616-622, doi: 10.1016/j.surg.2008.02.003, indexed in Pubmed: 18436009.

14. Malcic-Gürbüz J, Akalin A, Gümüşcü $B$, et al. Clinical implications of concomitant variations of the testicular, suprarenal and renal veins: a case report. Ann Anat. 2002; 184(1): 35-39, indexed in Pubmed: 11876480.

15. Merklin R, Mitchels NA. The variant renal and suprarenal blood supply with data on the inferior phrenic, ureteral and gonadal arteries. J Int Coll Surg. 1958; 29: 41-76.

16. Mishra GP, Bhatnagar S, Singh B. Unilateral triple renal veins and bilateral double renal arteries: a unique case report. Int J Anat Res. 2014; 2: 239-241.

17. Moore KL, Persaud TVN. The Developing Human: Clinically Oriented Embryology, 8th Ed. Saunders, Philadelphia 2007.

18. Nayak BS. Multiple variations of the right renal vessels. Singapore Med J. 2008; 49(6): e153-e155, indexed in Pubmed: 18581008.

19. Nayak SB, Sirasanagandla SR, Shetty SD, et al. Multiple vascular variations at the vicinity of the left kidney. Anat Sci Int. 2013; 88(4): 230-233, doi:10.1007/s12565-0130177-y, indexed in Pubmed: 23649479.

20. Ozkan U, Oğuzkurt L, Tercan F, et al. Renal artery origins and variations: angiographic evaluation of 855 consecutive patients. Diagn Interv Radiol. 2006; 12(4): 183-186, indexed in Pubmed: 17160802.

21. Petru B, Elena S, Dan I, et al. The morphology and the surgical importance of the gonadal arteries originating from the renal artery. Surg Radiol Anat. 2007; 29(5): 367-371, doi: 10.1007/s00276-007-0224-2, indexed in Pubmed: 17593308.

22. Saldarriaga B, Pinto S, Ballesteros L. Morphological expression of the renal artery: a direct anatomical study in a colombian half-caste population. Int J Morphol. 2008; 26(1): 31-38, doi: 10.4067/s0717-95022008000100005.

23. Sampaio F, Aragão A. Anatomical relationship between the renal venous arrangement and the kidney collecting system. J Urol. 1990; 144(5): 1089-1093, doi: 10.1016/ s0022-5347(17)39665-9. 
24. Satyapal KS. Classification of the drainage patterns of the renal veins. J Anat. 1995; 186 ( Pt 2): 329-333, indexed in Pubmed: 7649831.

25. Satyapal KS, Haffejee AA, Singh B, et al. Additional renal arteries: incidence and morphometry. Surg Radiol Anat. 2001; 23(1): 33-38, indexed in Pubmed: 11370140.

26. Satyapal KS, Kalideen JM, Haffejee AA, et al. Left renal vein variations. Surg Radiol Anat. 1999; 21(1): 77-81, indexed in Pubmed: 10370998.

27. Senecail B, Bobeuf J, Forlodou P, et al. Two rare anomalies of the left renal vein. Surg Radiol Anat. 2003; 25(5-6): 465-467, doi: 10.1007/s00276-003-0164-4, indexed in Pubmed: 13680187.

28. Smithuis TH. The problem of renal segmentation in connection with the modes of ramification of the renal artery and the renal vein. Arch Chir Neerl. 1956; 8(3): 227-241, indexed in Pubmed: 13373589.

29. Standring S. Gray's Anatomy: The Anatomical Basis of Clinical Practice, 40th Ed. Churchil Livingstone, Spain 2008.

30. Tank PW, P W. Grant's Dissector, 15th Ed. Lippincott Williams and Wilkins, Philadelphia 2012.
31. Tanyeli E, Uzel M, Soyluoğlu Al. Complex renal vascular variation: a case report. Ann Anat. 2006; 188(5): 455-458, doi: 10.1016/j.aanat.2006.05.013, indexed in Pubmed: 16999210.

32. Uzmansel D, Ozturk NC, Kara A, et al. A rare combination of vascular variations of both kidneys. Surg Radiol Anat. 2014; 36(2): 195-198, doi:10.1007/s00276-013-1134-0, indexed in Pubmed: 23661307.

33. Vatsala AR, Ajay KT, Mavishettar GF, et al. A study on branching pattern of renal arteries. Int J Anat Res. 2014; 2: 270-272.

34. Yi SQ, Ueno $Y$, Naito $M$, et al. The three most common variations of the left renal vein: a review and metaanalysis. Surg Radiol Anat. 2012; 34(9): 799-804, doi: 10.1007/s00276-012-0968-1, indexed in Pubmed: 22535303.

35. Zhu J, Zhang L, Yang Z, et al. Classification of the renal vein variations: a study with multidetector computed tomography. Surg Radiol Anat. 2015; 37(6): 667-675, doi: 10.1007/s00276-014-1403-6, indexed in Pubmed: 25567101. 